
Military Technical College, Kobry El-Kobbah, Cairo, Egypt

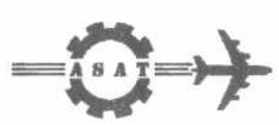

$9^{\text {th }}$ International Conference

On Aerospace Sciences \&

Aviation Technology

\title{
ANALYSIS OF THE ANGLE TRACKING ERROR \\ INDUCED IN A RADAR HOMING MISSILE \\ BY PHASE-FRONT DISTORTED JAMMING
}

\author{
ALADIN ASSISI*
}

\section{ABSTRACT}

In this paper a general analytical formula for the error induced in an angle tracking system by a phase front distorted jamming is derived. The electric fields originating from two coherent jamming sources are analyzed. The amplitude and phase of their resultant are computed; from which the parameters affecting that field are studied and the phase front equation is derived and plotted for different values of those parameters. Further analysis of the phase front equation leads to studying its inclination to the ideal spherical phase front, which leads to a general formula for the induced angular error. A similar approach was adopted in ref. [5] but the derivation was done in a special case. It is interesting that the resulting angular error depends only on the jamming system parameters and does not depend on the tracking system parameters or even on its type.

\section{KEY WORDS}

Proportional navigation, self-protection jamming, phase front, phase-front distortion, cross-eye, angle deception technique, coherent jamming sources,

\section{NOMENCLATURE}

Angular tracking error $\left(\beta_{j}\right)$, real and measured target-viewing angles $\left(\lambda_{r}\right.$ and $\left.\lambda_{m}\right)$, Target-Missile relative velocity $\left(\boldsymbol{V}_{\boldsymbol{r}}\right)$, lateral separation $\boldsymbol{d}$, ratio between jamming wave amplitudes $(\gamma)$, intentional phase difference $\varphi_{12}()$ and the path phase difference between the two waves $(\psi)$.

\footnotetext{
- PhD., Egyptian Armed Forces
} 


\section{INTRODUCTION}

Since the phase front of the wave scattered from a target is a sphere centered at the target, the stable equilibrium position of any reliable angle tracking system has to be perpendicular to that phase front [4]. The goal of a self-protection jamming system is to induce an error $\left(\beta_{j}=\lambda_{m}-\lambda_{r}\right)$ in the equilibrium position of the angle tracking system of a radar-guided missile; resulting in an error of measuring the target-viewing angle $(\lambda)$ shown in Fig. 1.

If the self-protection system generates a wave whose phase front is not perpendicular to the missile-target line; it imposes an error in the equilibrium position of any angle tracking system to be used by the missile seeker without need to get any information about its characteristics. This is the basic idea upon which the phase front distortion technique is based. This angle deception technique, commonly known as "cross-eye" technique, is applicable to any active, semi-active or passive radar homing or tracking system $[5,6]$.

This technique utilizes two coherent jamming sources $\left(\boldsymbol{J}_{1}\right.$ and $\left.\boldsymbol{J}_{2}\right)$ installed at the extremities of the target $\mathbf{T}$ at a lateral separation $\boldsymbol{d}[\mathrm{m}]$, tuned to the missile seeker frequency $f_{c}$. The ratio between their powers is $\gamma^{2}$ and an intentional phase difference $\varphi_{12}$ is imposed between them. We shall prove that this configuration results in a phase-front distorted wave that affects the stable equilibrium position of the missile seeker. We shall also derive a general analytical expression for the induced angular error.

\section{ANALYSIS OF PHASE FRONT DISTORTION EFFECT}

Figure 1 shows some basic geometric relationships necessary to analyze the effect of phase front distortion on the performance of a homing missile seeker. In this figure we can see the following:

a. The missile and target center coordinates are $\left(x_{M}, y_{M}\right)$ and $\left(x_{T}, y_{T}\right)$ respectively.

b. The target body is slanted by an angle $h_{t}$ with respect to the negative x-axis.

c. The missile-target center distance is $R_{T M}$.

d. The distance between the missile $M$ and the first source $J_{1} R_{M J 1}$ is smaller than $R_{T M}$ by $(d / 2)^{*} \sin \left(\lambda+h_{t}\right)$, while that between it and $J_{2} R_{M J 2}$ is longer than $R_{T M}$ by the same amount.

$$
\begin{aligned}
& R_{M J 1}=R_{T M}-(\mathrm{d} / 2)^{*} \sin \left(\lambda+h_{t}\right) \\
& R_{M J 2}=R_{T M}+(\mathrm{d} / 2)^{*} \sin \left(\lambda+h_{t}\right)
\end{aligned}
$$

e. The missile sees $J_{1}$ slanted by an angle $\alpha_{j} / 2$ to the right of the target center and $J_{2}$ slanted to the left by the same angle, where $\alpha_{j}$ is the angular difference between the two jamming antennas from the missile's point of view. We shall call it the jamming sources' resolution angle. 


$$
\alpha_{j}=d^{*} \cos \left(\lambda+h_{t}\right) / R_{T M} \quad[\mathrm{rad}]
$$

If $\boldsymbol{J}_{1}$ leads $\boldsymbol{J}_{2}$ in phase by $\boldsymbol{\varphi}_{12}$ and their amplitude ratio $\gamma$ is slightly less than 1 ; we can describe the electric field resulting from $J_{1}$ at the missile receiver by [5]:

$$
\begin{aligned}
E_{1} & =\gamma E_{0} e^{j\left[2 \pi f t-(2 \pi / l)\left(R_{T M}-(d / 2) \sin \left(\lambda+h_{t}\right)\right)\right]} \\
& =\gamma E_{0} e^{j\left[2 \pi f t-\left(2 \pi R_{T M} / l\right)+(\psi / 2)\right]}
\end{aligned}
$$

where $E_{0}$ is the field strength amplitude due to $\mathbf{J} 2$ at the missile location $I$ is the wave length

$\psi$ is the path phase difference between the two waves.

$$
\psi=\frac{2 \pi d \cdot \sin \left(\lambda+h_{t}\right)}{l}
$$

We can describe the electric field resulting from $J_{2}$ at the missile receiver by [5]:

$$
\begin{aligned}
E_{2} & =E_{0} e^{j\left[2 \pi f t-(2 \pi / l)\left(R_{T M}+(d / 2) \sin \left(\lambda+h_{t}\right)\right)-\varphi_{12}\right]} \\
& =E_{0} e^{j\left[2 \pi f t-\left(2 \pi R_{T M} / l\right)-(\psi / 2)-\varphi_{12}\right]}
\end{aligned}
$$

The resultant field will be:

$$
E_{J}=E_{0} e^{j\left[2 \pi f t-\left(2 \pi R_{T M} / t\right)\right]} \cdot\left[\gamma e^{j(\psi / 2)}+e^{-j\left((\psi / 2)+\varphi_{12}\right)}\right]
$$

The effective phase difference between the two waves at the missile receiver is:

$$
\varphi_{e f f}=\psi+\varphi_{12}=\left(\frac{2 \pi d \sin \left(\lambda+h_{t}\right)}{l}\right)+\varphi_{12}
$$

From (7) the resultant field amplitude is:

$$
\left|E_{J}\right|=E_{0} \cdot \sqrt{\left(\gamma+\cos \left(\varphi_{e f f}\right)\right)^{2}+\sin ^{2}\left(\varphi_{e f f}\right)}=\sqrt{\gamma^{2}+2 \gamma \cos \left(\varphi_{e f f}\right)+1}
$$

And its phase is:

$$
\varphi_{J}=\frac{2 \pi R_{T M}}{l}+\arctan \left(\frac{\gamma \sin (\psi / 2)-\sin \left[(\psi / 2)+\varphi_{12}\right]}{\gamma \cos (\psi / 2)+\cos \left[(\psi / 2)+\varphi_{12}\right]}\right)
$$


The variation of the resultant field relative amplitude $\left(E / E_{0}\right)$ is plotted vs. all possible unambiguous values of $\lambda$ in Fig.2. In Fig.3, $\lambda$ is varied between $-10^{\circ}$ and $+10^{\circ}$ at different values of $\gamma$ between 0.7 and 0.95 . Both the relative amplitude $\left(E_{J} / E_{0}\right)$ and relative phase $\varphi_{r}=\varphi_{J}-\frac{2 \pi R_{T M}}{l}$ are plotted according to (9) and (10), respectively for a lateral separation between the two sources $\boldsymbol{d}=\mathbf{5 l}$ and an intentional phase shift $\varphi_{12}=180^{\circ}$. It is evident that the resultant field phase is reversed at the directions of minimum field amplitude that repeat periodically. From (10) we can deduce the equation of constant phase contours; which is the
equation of the phase front:

$$
r=\left(\frac{l}{2 \pi}\right) \cdot\left[\varphi_{J}-\arctan \left(\frac{\gamma \sin \left(\frac{\psi}{2}\right)-\sin \left[\left(\frac{\psi}{2}\right)+\varphi_{12}\right]}{\gamma \cos \left(\frac{\psi}{2}\right)+\cos \left[\left(\frac{\psi}{2}\right)+\varphi_{12}\right]}\right)\right]
$$

Fig. $4 \mathbf{a}$ and $\mathbf{b}$ show consecutive phase fronts according to (11) for $d=51$ and $\gamma=0.5$ and 0.9 respectively. The original spherical phase fronts are plotted in dashed lines for reference. It can be noticed from this figure that the distorted phase front is inclined to the circular one by an error angle $\beta_{j}$ that increases with $\gamma$, varies with $\lambda$ but does not vary with $\mathbf{r}$. The relation between $\beta_{j}$ and $\lambda$ can be derived as follows:

If we move on an undistorted circular phase front with radius $r$ an infinitesimal distance $r \delta \lambda$; the phase of the distorted wave changes by $\delta \varphi$. In order to return to the original phase we need to move a radial distance $\delta$ and arrive at another circular phase front that differs from the first one by $\delta \varphi$. Whence; the inclination of the distorted phase front on the circular one can be expressed as

$$
\tan \left(\beta_{j}\right)=\frac{d r}{r d \lambda}=\left(\frac{1}{r}\right)\left(\frac{d r}{d \lambda}\right)
$$

Looking at (5) and (10) we notice that $\varphi_{\mathrm{j}}$ is a function of two main variables $r$ and $\lambda$; assuming both $\gamma$ and $\varphi_{12}$ are constant. We can express it as a sum of two separable
functions of two independent variables:

$$
\varphi_{J}(r, \lambda)=\varphi_{c}(r)+\varphi_{r}(\lambda)
$$

Where the phase due to the wave propagation between the missile and the target
center is independent of $\lambda$.

$$
\varphi_{c}(r)=\frac{2 \pi r}{l}
$$


and the relative phase difference between the jamming wave and the reflected one is independent of $r$.

$$
\varphi_{r}=\arctan \left(\frac{\gamma \sin (\psi / 2)-\sin \left[(\psi / 2)+\varphi_{12}\right]}{\gamma \cos (\psi / 2)+\cos \left[(\psi / 2)+\varphi_{12}\right]}\right)
$$

Investigating (14); we can write:

$$
\begin{aligned}
d \varphi_{J}(r, \lambda)= & d \varphi_{c}(r)+d \varphi_{r}(\lambda) \\
& =\left(\frac{d \varphi_{c}}{d r}\right) d r+\left(\frac{d \varphi_{r}}{d \lambda}\right) d \lambda \\
& =\left(\frac{d \varphi_{J}}{d r}\right) d r+\left(\frac{d \varphi_{J}}{d \lambda}\right) d \lambda
\end{aligned}
$$

By definition; the phase is constant on the phase front. Therefore; equating both sides of (17) with zero we get:

$$
\begin{aligned}
& \left(\frac{d \varphi_{c}}{d r}\right) d r=-\left(\frac{d \varphi_{r}}{d \lambda}\right) d \lambda \\
& \therefore\left(\frac{d r}{d \lambda}\right)=-\left(\frac{l}{2 \pi}\right) \cdot\left(\frac{d \varphi_{r}}{d \lambda}\right)
\end{aligned}
$$

It remains only to differentiate (16). We can rewrite it as:

$$
\begin{aligned}
& \tan \left(\varphi_{r}\right)=\left(\frac{\gamma \sin (\psi / 2)-\sin \left[(\psi / 2)+\varphi_{12}\right]}{\gamma \cos (\psi / 2)+\cos \left[(\psi / 2)+\varphi_{12}\right]}\right) \\
& \therefore \sec ^{2}\left(\varphi_{r}\right) d \varphi_{r}=\frac{d}{d \psi}\left(\frac{\gamma \sin \left(\frac{\psi}{2}\right)-\sin \left[\left(\frac{\psi}{2}\right)+\varphi_{12}\right]}{\gamma \cos \left(\frac{\psi}{2}\right)+\cos \left[\left(\frac{\psi}{2}\right)+\varphi_{12}\right]}\right) \cdot \frac{2 \pi d \cos \left(\lambda+h_{t}\right)}{l} d \lambda
\end{aligned}
$$

Differentiating; the R.H.S. arrives at:

$$
R \cdot H \cdot S \cdot=\left(\frac{\left(\gamma^{2}-1\right)\left(\frac{\pi d \cos \left(\lambda+h_{i}\right)}{l}\right) d \lambda}{\left[\gamma \cos (\psi / 2)+\cos \left[(\psi / 2)+\varphi_{12}\right]\right]^{2}}\right)
$$

And the L.H.S. arrives at: 
L.H.S. $=\left(\frac{\left(\gamma^{2}+2 \gamma \cos \left(\psi+\varphi_{12}\right)+1\right) d \varphi_{r}}{\left[\gamma \cos (\psi / 2)+\cos \left[(\psi / 2)+\varphi_{12}\right]\right]^{2}}\right)$

Equating the two sides we get:

$\frac{d \varphi_{r}}{d \lambda}=\left(\frac{\pi \cdot d \cdot \cos \left(\lambda+h_{t}\right)}{l}\right) \cdot\left(\frac{\gamma^{2}-1}{\gamma^{2}+2 \gamma \cos \left(\psi+\varphi_{12}\right)+1}\right)$

Substituting in (17); we get the differential equation of the distorted phase front:

$$
\begin{aligned}
& \frac{d r}{d \lambda}=-\left(\frac{l}{2 \pi}\right)\left(\frac{\pi \cdot d \cdot \cos \left(\lambda+h_{t}\right)}{l}\right) \cdot\left(\frac{\gamma^{2}-1}{\gamma^{2}+2 \gamma \cos \left(\psi+\varphi_{12}\right)+1}\right) \\
& \frac{d r}{d \lambda}=-\left(\frac{d \cdot \cos \left(\lambda+h_{t}\right)}{2}\right) \cdot\left(\frac{\gamma^{2}-1}{\gamma^{2}+2 \gamma \cos \left(\varphi_{e f f}\right)+1}\right)
\end{aligned}
$$

Substituting in (13); we get the value of error angle:

$$
\begin{aligned}
& \tan \left(\beta_{j}\right)=\left(\frac{d \cos \left(\lambda+h_{t}\right)}{2 r}\right) \cdot\left(\frac{1-\gamma^{2}}{\gamma^{2}+2 \gamma \cos \varphi_{e f}+1}\right) \\
& \therefore \tan \left(\beta_{j}\right)=\left(\frac{\alpha j}{2}\right) \cdot\left(\frac{1-\gamma^{2}}{\gamma^{2}+2 \gamma \cos \varphi_{e f}+1}\right)
\end{aligned}
$$

where

$$
\begin{aligned}
& \alpha_{j}=d \cos \left(\lambda+h_{t}\right) / R_{T M} \\
& \varphi_{\text {eff }}=\psi+\varphi_{12}=\left(\frac{2 \pi d \sin \left(\lambda+h_{t}\right)}{l}\right)+\varphi_{12}
\end{aligned}
$$

\section{CONCLUSION}

The equation (22) is the general formula for computing the error generated by phase front distortion in any angle tracking system; regardless of its type and characteristics. Equations (23) and (24) determine the dependencies of the resolution angle $\alpha_{j}$ and the effective phase difference between the two jamming sources $\varphi_{\text {eff }}$ on different parameters of the jamming problem; such as the angle of sight $\lambda$, the target 
inclination $\boldsymbol{h}_{\boldsymbol{t}}$, the wavelength $\boldsymbol{I}$ and the lateral distance $\boldsymbol{d}$. No reference is made to tracking system parameters. The results are completely independent of the angular tracking technique.

\section{REFERENCES:-}

[1] M.V. MAXIMOV, "Electronic Homing Systems", translated by W.F.Barton, Artech House, 1988.

[2] A. Golden, "Radar Electronic Warfare", AIAA Education Series, published by the American Institute of Aeronautics and Astronautics, Inc., 1987.

[3] A. ASSISI, "Simulating And Maximizing The Effect of Jamming On Proportional Navigation Homing Guidance", Proceedings of the $8^{\text {th }}$ ASAT Conference, May 1999.

[4] LEROY B. VAN BRUNT, “Applied ECM V.1”, EW Engineering, 1979.

[5] S. A. VAKIN and L. N. SHUSTOV, "Principles of Jamming and Electronic Reconnaissance", Soviet Radio, 1969. Translated by the American Intelligence in 1970.

[6] D.C. Schleher, "Electronic Warfare in Information Age", Artech House, 1999. 


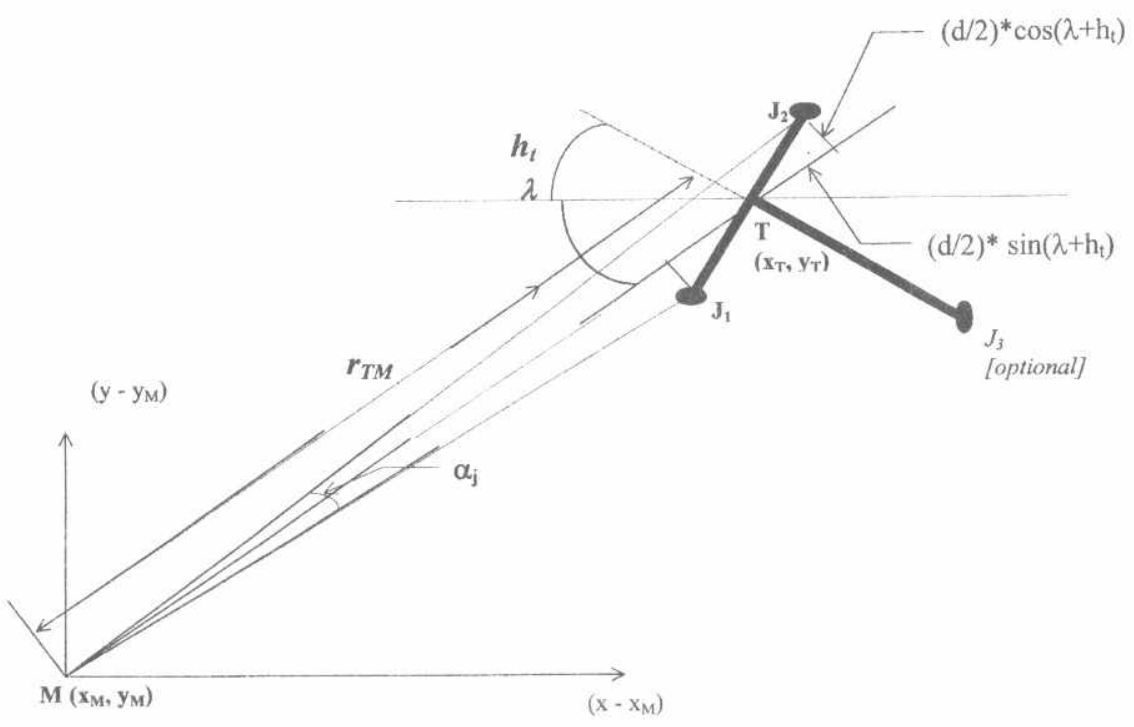

FIG.1 Basic Geometric Relationships

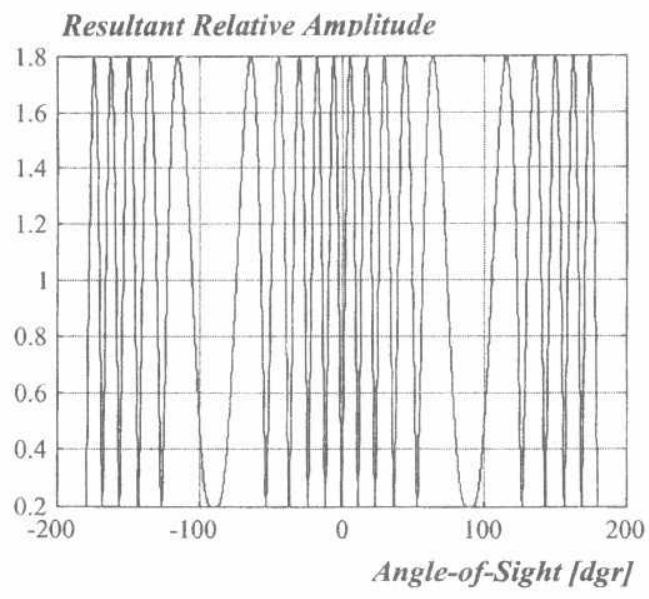

Fig.2 Variation of Relative Field Amplitude with Viewing Angle 


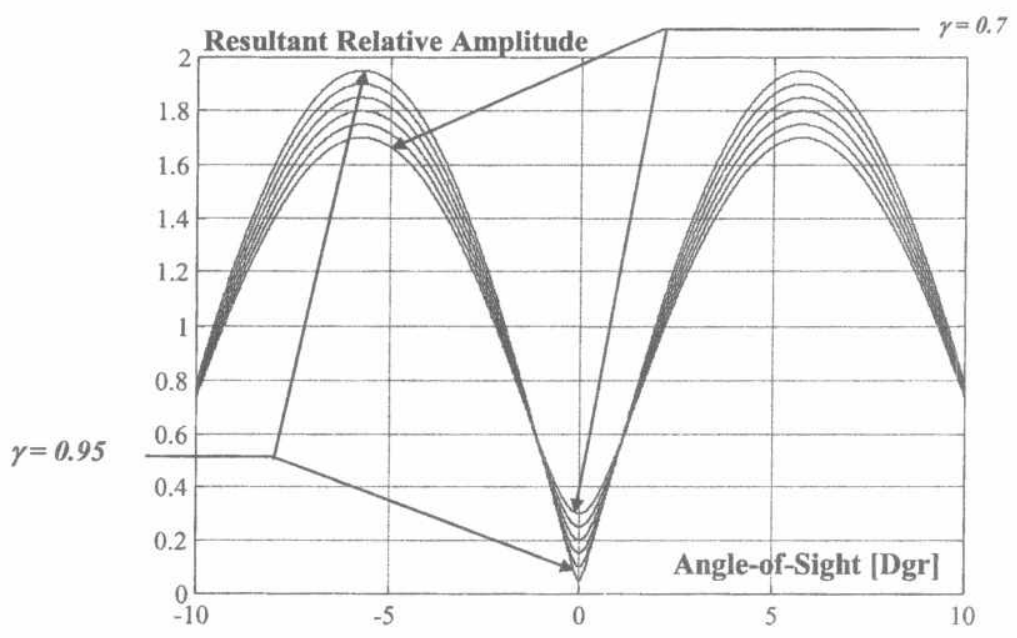

Fig. 3a Variation of Relative Field Amplitude with Viewing Angle

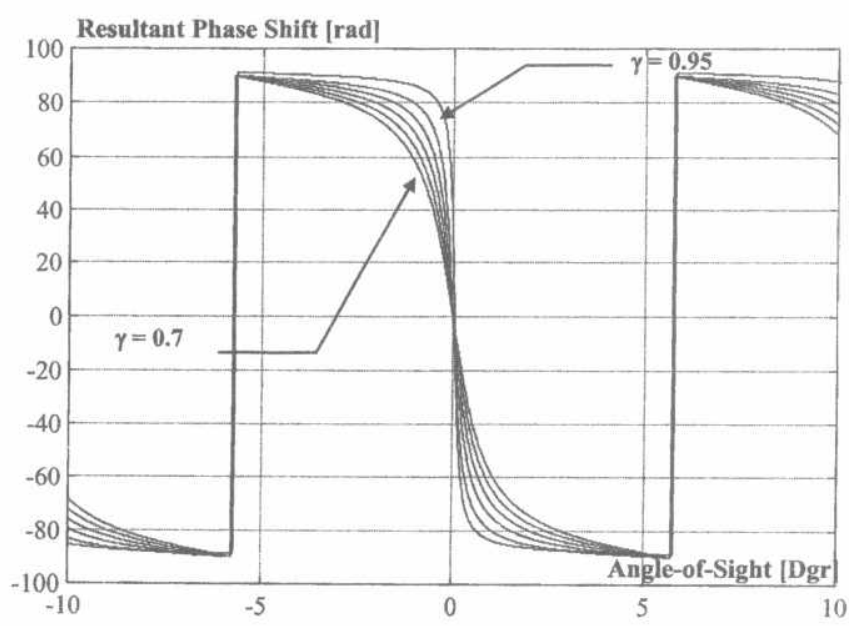

Fig. 3b Variation of Relative Field Phase with Viewing Angle 


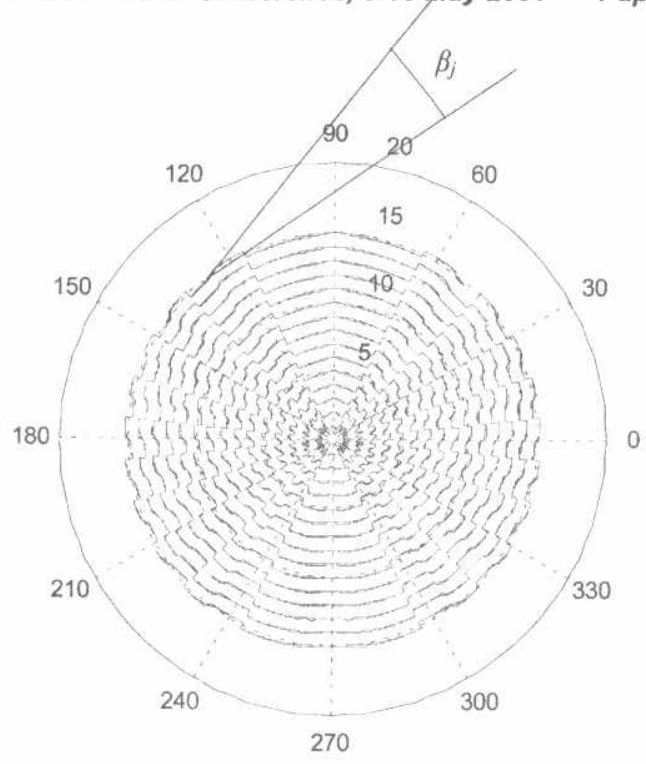

a. $d=5 l$ and $y=0.5$

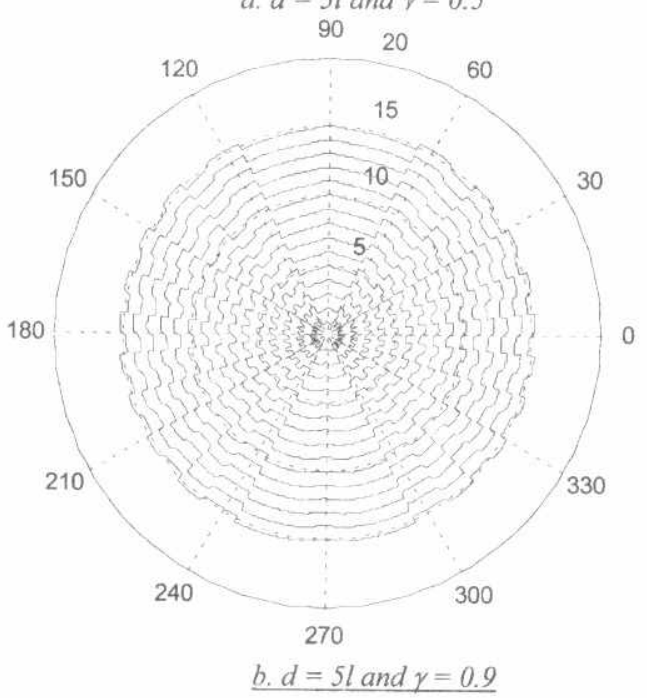

Fig. 4 Subsequent Distorted Phase Fronts

(in polar coordinates)

[Note that they are inclined on the dashed circular phase fronts and that the inclination $\beta_{j}$ increases with $\left.\gamma\right]$ 